\title{
Development of an Active Thermal Louver for CubeSats Controlled via SMA Actuator
}

\author{
Dario Franchin \\ Department of Industrial Engineering (DII) \\ University of Padova \\ Padova, Italy \\ dariofranchin.df@gmail.com
}

\author{
Florian Schummer \\ Chair of Astronautics (LRT) \\ Technical University of Munich \\ Munich, Germany \\ f.schummer@tum.de
}

\begin{abstract}
In recent years, CubeSats proved to be valuable resources both for commercial and scientific purposes, leading to a significant technological development in terms of payloads and on-board instrumentation. The employment of more advanced technology usually implies a higher power consumption, and a consequently increased amount of waste heat. The typical, passive thermal control systems currently employed on small satellites, such as paints and coatings, may not be sufficient to guarantee a proper thermal stability, and therefore more elaborate and efficient systems are required. Based on the actuator IRESA (Intelligent Redundant Spacecraft Actuator), under development at the Chair of Astronautics of the Technical University of Munich, a new design for a compact, reliable, active thermal control system for CubeSats is proposed. IRESA is a shape-memory-alloy-based, low-power-consuming, high-forceper-unit-mass actuator embedded on a PCB, compatible with the lateral panel of a $1 \mathrm{U}$ CubeSat. IRESA produces a linear displacement of $3.5 \mathrm{~mm}$ exploiting the contraction of redundant SMA wires heated efficiently through the Joule effect; the displacement can be converted into rotation, allowing the actuator to operate a variety of subsystems. The presented design for the TCS consists of an external louver moved by IRESA, capable of modifying the emissivity of a small radiator or regulate the power emission from the inner part of the satellite to space. The design of the louver was obtained studying the louvered surfaces employed over the last fifty years in larger satellites and adapting the geometry to the features of the actuator, with the general design driver of a minimum complexity for the assembly. Therefore, a configuration with a single blade was chosen and implemented; like its larger counterparts, it reaches and maintains every angular position between the fully closed and fully open states, performing a 90 degrees rotation; the linear displacement of the SMA wires is converted into rotation by a simple lever principle. The proposed subsystem meets the CubeSat Design Specification in terms of geometry and compatibility with a CubeSat of at least $2 \mathrm{U}$. The subsystem was developed as a master thesis project at the Chair of Astronautics of the Technical University of Munich starting from October, 2018. A prototype was successfully integrated in March, 2019, and good results were obtained during the first functional, vacuum chamber and vibration tests, during which the louver proved to work properly and continuously during the opening and closing procedures, and maintained its structural integrity.
\end{abstract}

Keywords-TCS, thermal control system, louver, CubeSat, SMA, shape memory alloys, spacecraft, technology

\section{SHAPE MEMORY ALLOYS AND IRESA}

\section{A. Physical properties of shape memory alloys}

Shape memory alloys (SMA) are a class of smart materials characterised by the ability to recover large induced shape deformations when undergoing a specific thermo-mechanical cycle. The most common variants of SMAs are nickel (Ni) and titanium (Ti) compounds with different percentages of the two metals, and the different alloys share the name of NiTinol [1].

The characteristic shape memory effect (SME) exhibited by shape memory alloys comes from the transformations occurring in the microscopic lattice when a change in the thermal and mechanical boundary conditions takes place. The SME displays as the material switches between three different stable phases: austenite, twinned martensite and de-twinned martensite [2].

In a stress $(\sigma)$ - strain $(\varepsilon)$ - temperature (T) space (Fig. 1), the thermo-mechanical cycle of the SME starts with the material in austenite form, which is the phase stable at higher temperature and characterised by an ordered, highly symmetrical lattice; following the curve, the subsequent formation of twinned martensite is achieved decreasing the temperature under no external load. Twinned martensite does not display macroscopical variations of shape if compared with austenite, due to the small rearrangements which occur in the microscopic lattice.

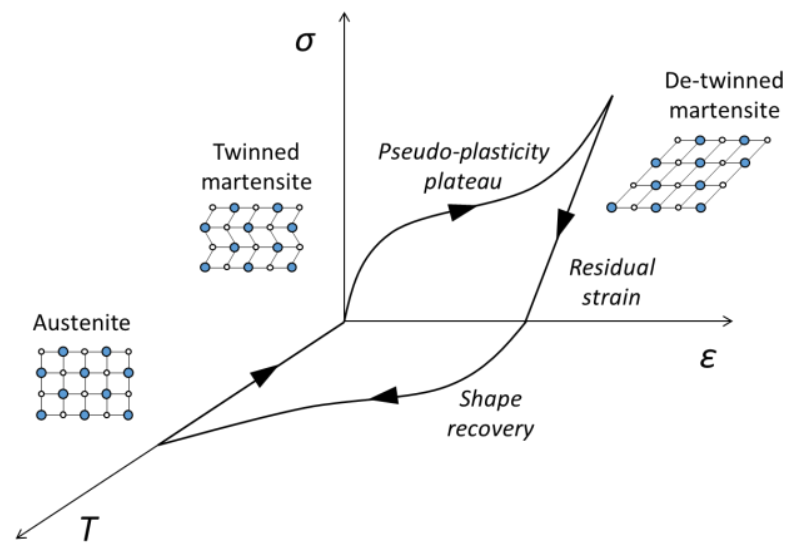

Fig. 1. The complete thermo-mechanical cycle of the shape memory effect, reworked from [2]. 


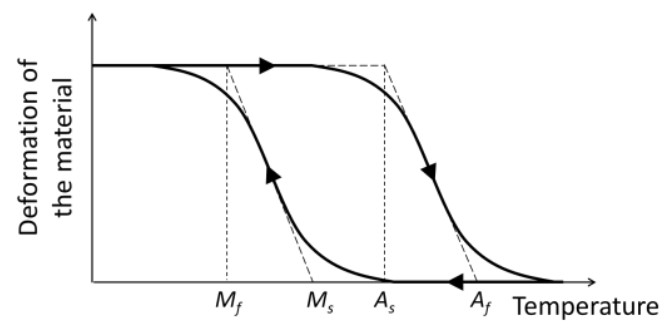

Fig. 2. The hysteresis curve associated with the SME with a highlight on the characteristic temperatures, reworked from [2].

Upon increasing the stress in the material, a first linear, elastic behavior is encountered, followed by a pseudo-plastic plateau in the $\varepsilon-\sigma$ plane. The plateau occurs at the detwinning of the martensite. It has a nearly constant stress level; when the de-twinning is complete, the material responds elastically to a further increase in the stress level up to the yield point. At the end of the plateau, the formerly twinned martensite is de-twinned, and a macroscopical deformation is found in the material. Upon unloading, the material retains the induced strain, and only an increase in the temperature causes a recovery of the original austenite structure, eliminating every induced shape variation. The process of de-twinning can also be achieved progressively as the temperature decreases while an external load is applied constantly on the SMA, thus avoiding a clear mid-phase represented by twinned martensite and switching directly between austenite and de-twinned martensite.

In this case, when operating at a constant stress level the material exhibits a hysteretic behavior during heating and cooling (Fig. 2). Therefore four characteristic temperatures can be defined for the material: $\mathrm{M}_{\mathrm{s}}$ (martensite start temperature), $\mathrm{M}_{\mathrm{f}}$ (martensite finish temperature), $\mathrm{A}_{\mathrm{s}}$ (austenite start temperature) and $\mathrm{A}_{\mathrm{f}}$ (austenite finish temperature). For temperatures between the extremes of the transformation different fractions of each phase are found in the material: a specific shape is therefore associated to a determined temperature level or range, offering possibilities to exploit the response to temperature variations in order to control the behavior of the SMA and to make it suitable to be the active component in actuators and sensors [3,4]. Strains up to $8 \%$ are possible for a sample of SMA, but lower values can be adopted as operative strain level in order to preserve the integrity of the material for a higher number of cycles, extending its possible service life.

\section{B. IRESA - Intelligent Redundant Spacecraft Actuator}

IRESA (Intelligent Redundant Spacecraft Actuator) is a compact, high force-per-unit-mass actuator operating with SMAs developed by the FGW Forschungsgemeinschaft Werkzeuge und Werkstoffe e. V. and the Chair of Astronautics (LRT) of the Technical University of Munich. The description of the actuator presented in this section is extracted and reworked from [5], in which the features of the hardware are presented in a more detailed way. IRESA makes use of NiTinol wires exhibiting the previously introduced SME: the wires are coupled with a return (bias) spring (Fig. 3), which provides the force necessary for the de-twinning process and the reset of the mechanism. The wires are in their de-twinned martensite state at room temperature, and they contract (passing to the austenite phase) when heated via the Joule effect. The actuator is embedded on a PCB and contained in an envelope of $80 \times 30 \times 8$ $\mathrm{mm}$, compatible with the side panel of a $1 \mathrm{U}$ CubeSat. The mechanism produces a linear displacement of $3.5 \mathrm{~mm}$ imposing a maximum strain of $3 \%$ to the wires. The mechanism is designed to reach an operative life of at least 5,000 actuations per wire.
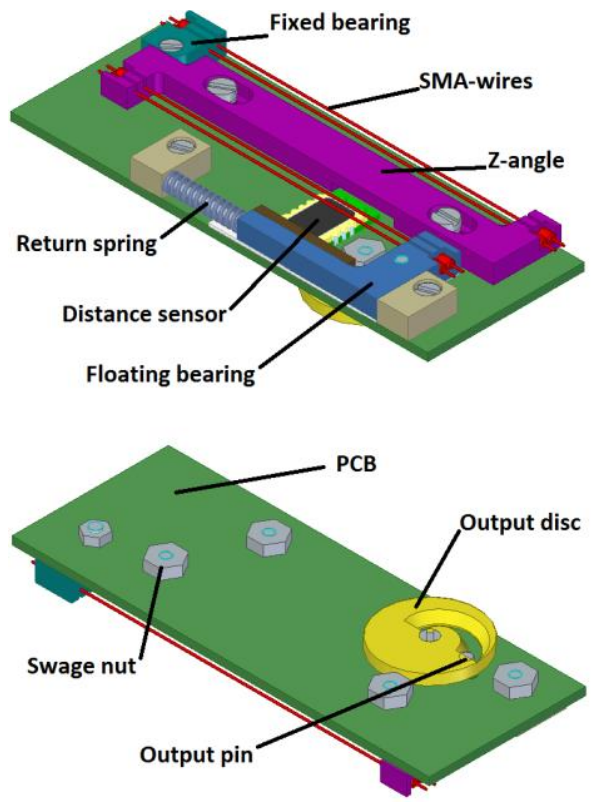

Fig. 3. The CAD simplified model of IRESA showing the main elements of the actuator; figure reported without modifications from original source [5].

The total length of the wires, corresponding to $131 \mathrm{~mm}$, splits into two shorter wires (red colour in figure 3) which contract simultaneously, moving an intermediate Z-shaped element (violet) and producing the nominal output at the floating bearing (blue). The output pin attached to the floating bearing exports the motion, and connects to an optional output disk (yellow) mounted on the back of the board. The linear stroke of the pin converts to 180 degrees rotation of the disk. The board features redundant wires close to the primary elements, which form pairs, and work as back up units in case of failure or to perform a smooth switch without interruption of service when wear is detected in the main wires.

The output pin can attain and maintain every intermediate position between the two extremes, and a precise control can be performed during operations maintaining the temperature between the extremes of the transformation via a pulse width modulation. A position sensor determines the displacement of the floating bearing with a precision of $10 \mu \mathrm{m}$; a lower precision is achievable when the conversion into rotation takes place, due to tribological issues between the pin and the PEEK disk: an angular precision of $\pm 4^{\circ}$ is possible with the current version of the board. The microcontroller MSP430 by Texas Instruments mounted on the board is characterized by low power consumption during operation, guaranteeing an extremely low idle power consumption for IRESA. Table I reports the main parameters of the actuator. 
TABLE I. IRESA DATASHEET; DATA RETRIEVED AND REPORTED FROM THE DATASHEET CONTAINED IN [5].

\begin{tabular}{|l|l|l|}
\hline Parameter & Value & Unit \\
\hline Mass & 22 & $\mathrm{~g}$ \\
\hline Idle power consumption & 125 & $\mathrm{~mW}$ \\
\hline Operative power consumption in vacuum & 400 & $\mathrm{~mW}$ \\
\hline $\begin{array}{l}\text { Operative power consumption under } \\
\text { laboratory conditions }\end{array}$ & 1800 & $\mathrm{~mW}$ \\
\hline Nominal output translation & 3.5 & $\mathrm{~mm}$ \\
\hline Nominal output rotation & 180 & $\mathrm{deg}$ \\
\hline Rated output force & 19 & $\mathrm{~N}$ \\
\hline $\begin{array}{l}\text { Reset time in vacuum at } 20^{\circ} \mathrm{C} \text { environment } \\
\text { temperature }\end{array}$ & 90 & $\mathrm{~s}$ \\
\hline $\begin{array}{l}\text { Reset time under laboratory conditions at } \\
20^{\circ} \mathrm{C} \text { environment temperature }\end{array}$ & 35 & $\mathrm{~s}$ \\
\hline
\end{tabular}

\section{THERMAL CONTROL SYSTEM AND LOUVERS}

The use of an active thermal control system could prove beneficial for CubeSats both in case of cryogenically cooled equipment and in case a narrow temperature range is required for the internal environment of the satellite [6]. The greater stability offered by a more elaborate thermal control would allow for more power consuming and waste-heat generating technology to be mounted on CubeSats for operative or testing purposes.

Thermal louvers are thermal control systems which operate in combination with high emissivity surfaces, of which they are able to regulate the performance depending on the evolution of the internal temperature over time. They achieve this effect by means of movable external blades, which create a variable obtrusion to the power radiation with the external environment [7]. The main parameter used to characterize a louver and its effect on the radiator is its effective emissivity $\varepsilon_{\text {eff }}$, defined as:

$$
\varepsilon_{e f f}=\frac{q_{\text {out }}}{\sigma_{s b} A T^{4}}
$$

Effective emissivity correlates the amount of power $q_{\text {out }}$ [W] emitted by a generic surface with the power emitted by an equivalent black surface with an equal area $A\left[\mathrm{~m}^{2}\right]$ and at the same temperature $T[\mathrm{~K}]$, and it is therefore an indicator of the efficiency of the heat transfer $\left(\sigma_{\mathrm{sb}}\left[\mathrm{W} / \mathrm{m}^{2} \mathrm{~K}^{4}\right]\right.$ is the StefanBoltzmann constant). This parameter changes following the opening angle of the blades of the louver, with typical values of 0.08-0.1 for completely closed blades and $0.6-0.7$ for a maximum heat dissipation configuration, i.e. blades open at 90 degrees [8].

The support structure and the aspect ratio of most louver systems have a strong impact on the effective emissivity, reducing the emission capabilities even in maximum heat rejection mode. The efficiency of the TCS is therefore partially impaired by the presence of the louver. Opposed to the classical geometry consisting in an array of blades, in the case of this subsystem a simpler configuration with a single moving panel was adopted and developed (Fig. 4) in order to a) maximize the radiation capability of the system reducing the number of obtruding elements and b) reduce the mechanical complexity in order to match the features of IRESA, which is designated as the only source of actuation of the system. The adopted geometry is able to guarantee a good power dissipation when the louver is open while still allowing for the maximum insulation in case the blade is completely closed.

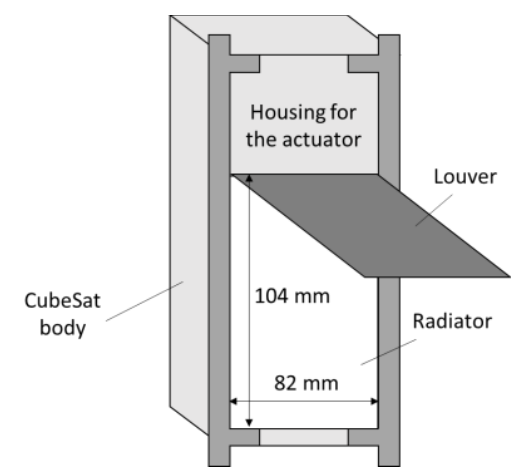

Fig. 4. A scheme of the preliminary design of the subsystem, representing the general structure of a $2 \mathrm{U}$ CubeSat.

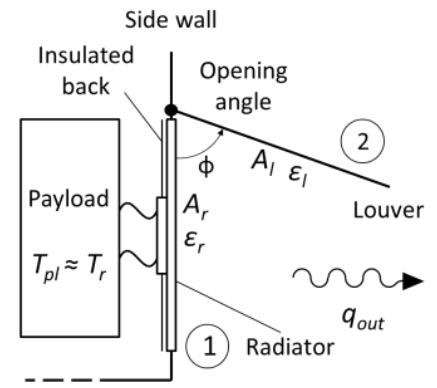

Fig. 5. The simplified thermal model accounting for the mutual interaction of the radiator and the louver.

The preliminary thermal analysis performed on the system is addressed to finding the potential operative range of the louver in terms of effective emissivity, as presented in (1) and in absence of external heat sources such as the solar power or the emission and albedo from the Earth surface. A simplified thermal model is implemented in MATLAB and two thermal nodes are considered: 1) the radiator and 2) the louver (Fig. 5); the two nodes are structurally decoupled and can only exchange heat through radiation, therefore parasitic conduction phenomena are neglected.

Future validations performed via experiments in a vacuum chamber could improve the model and verify the effect of the simplifications on its functioning. The performance of the system is assessed for a variation of the opening angle of the blade $\Phi$ (in the range 0-90 degrees) and for different values of the thermo-optical properties of the surfaces, respectively:

- $\varepsilon_{r}$, emissivity of the radiator in the infrared spectrum;

- $\varepsilon_{l}$, emissivity of the louver in the infrared spectrum;

The temperature of the radiator $T_{r}$ is set at a sensible value of $15^{\circ} \mathrm{C}$ for every simulation. A hypothesis of isothermy 
between the radiator and the payload is made, synonym of a perfect thermal connection between the bodies, in order to reduce the complexity of the model. The size of the radiator and the louver in terms of areas $A_{r}$ and $A_{l}$ is the same as the one reported in figure 4 , and it is derived from the standard size of a 2 U CubeSat as regulated by the CubeSat Design Standard [9] and considering the size of IRESA as part of the side panel.

The presented plots (Fig. 6 and 7) are obtained for a variation of the emissivity of the radiator $\left(\varepsilon_{r}\right)$ and the louver $\left(\varepsilon_{l}\right)$. The preliminary results show a good capability of the system in decoupling the radiator from space in the fully closed condition $\left(\varepsilon_{\text {eff }}\right.$ below 0.1 for each value of $\varepsilon_{r}$ ) while allowing a power dissipation close to the one achieved by a non-protected radiator in the fully open condition; on the other hand, it is observed that in order to achieve a better insulation when the blade is closed the value of $\varepsilon_{l}$ should be chosen as low as possible ( $\varepsilon_{e f f}$ approximately or below 0.1 when $\varepsilon_{l}$ is below 0.15 ), at least on the inner surface of the blade.

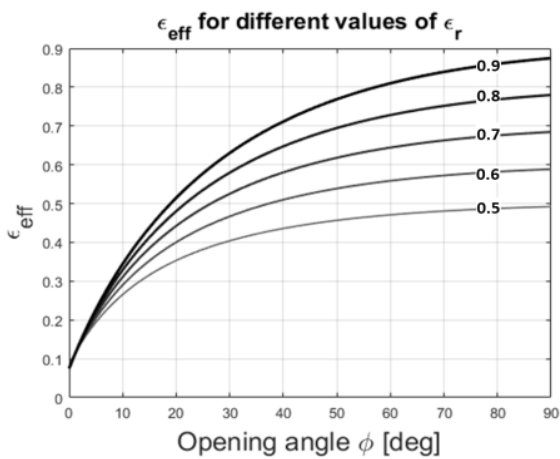

Fig. 6. The variation of $\varepsilon_{e f f}$ with the opening angle of the louver $\Phi$ for different emissivities $\varepsilon$

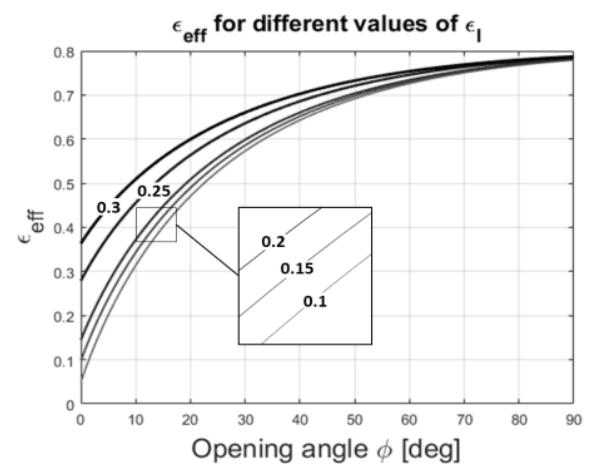

Fig. 7. The variation of $\varepsilon_{e f f}$ with the opening angle of the louver $\Phi$ for different emissivities $\varepsilon_{l}$.

\section{MECHANICAL DESIGN}

In order to guarantee the functioning of the system, a range of motion of 90 degrees must be produced by the mechanical connection between the louver and the actuator. A lever system is adopted to produce the rotation: a rigid connector is attached between the floating bearing of IRESA and the hinges of the louver, and it is designed in order to operate with a stroke $d$ of $3 \mathrm{~mm}$ (Tab. II), accounting for some margin over the total stroke of the actuator. The lever is designed in order to avoid the occurrence of any mechanical singularity during the complete cycle, condition which may cause a locking of the blade or an increase in the internal forces of the system. The angular position of the louver is directly driven by the translation of the output pin (Fig. 8), but a torsional spring mounted on one of the hinges of the louver guarantees a reinforcement to the design, and generates an extra preload helpful in maintaining the blade at the desired angle (Fig. 9). The structural elements of the prototype are built with 6061-T6 aluminum, while polyether ether ketone (PEEK) is used for the connection elements between IRESA and the moving panel as well as for the hinges of the system.

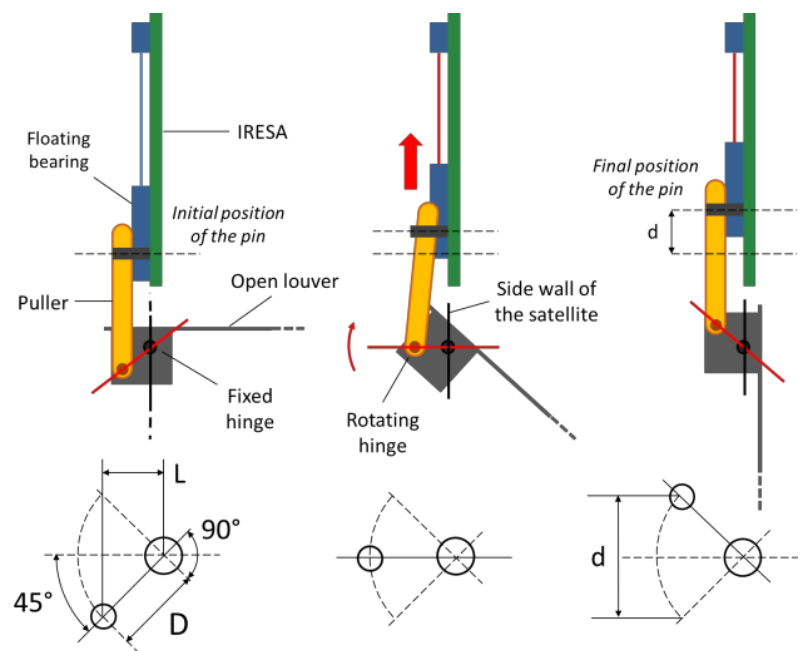

Fig. 8. A schematic working principle of the mechanics of the louver, showing a side view of the lever and the relative position of the hinges for three different configurations.

TABLE II. MAIN DIMENSIONS OF THE LEVER

\begin{tabular}{|c|c|c|}
\hline Quantity & Value & Unit \\
\hline $\mathrm{D}$ & 2.12 & $\mathrm{~mm}$ \\
\hline $\mathrm{L}$ & 1.5 & $\mathrm{~mm}$ \\
\hline $\mathrm{d}$ & 3 & $\mathrm{~mm}$ \\
\hline
\end{tabular}

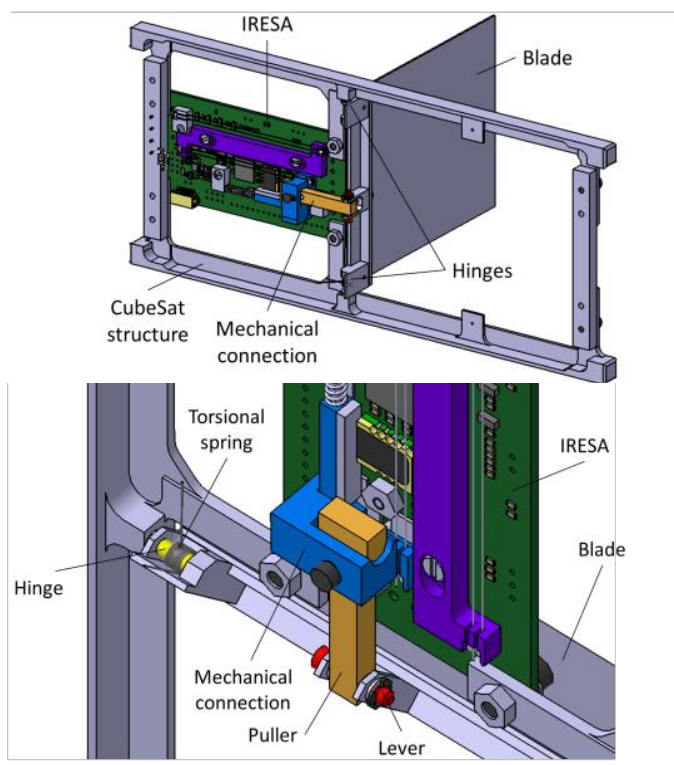

Fig. 9. The final design of the system, used to manifacture the first prototype. 


\section{PROTOTYPE INTEGRATION AND TESTING}

The final design adopted for the mechanism was manifactured at the workshops of the Technical University of Munich, and successfully integrated in order to prove the absence of defects in the geometry of the system. Due to a fast production process, some of the tolerances on the structural elements were not matched properly, causing the louver to interfere with the fillets of the structure and to remain at an angle of approximately 80 degrees when fully open, instead of the nominal 90 degrees (Fig. 10). The first functional test consisted in verifying the correct closing and opening operations, and it was carried out by running a simple contraction-relaxation sequence of IRESA.

The results of the test (Fig. 11) prove that the lever operates correctly over the nominal range of motion, allowing for a smooth rotation of the panel. The plot shows that a final angle of approximately 5 degrees was reached before the opening procedure: this result is due to the non-complete contraction of IRESA under laboratory conditions, where the power and the time necessary to achieve a complete contraction are too elevated due to convective heat transfer between the hot wire and the surrounding air at room temperature. Tests run in a vacuum chamber are expected to remove this trend from the behaviour of the system and exhibit a complete closing of the panel.

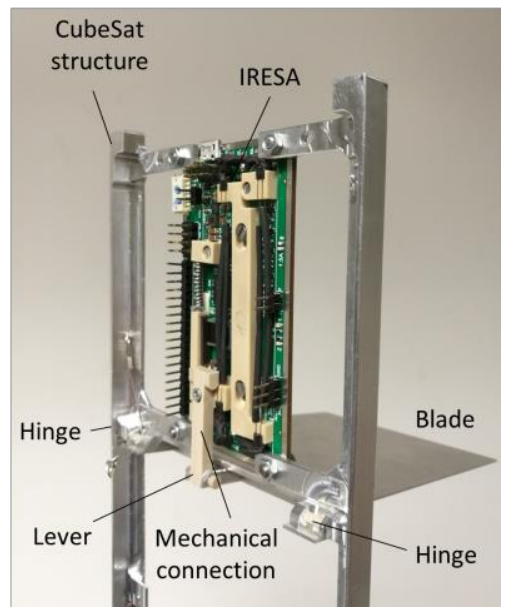

Fig. 10. The mechanical prototype of the system after integration.

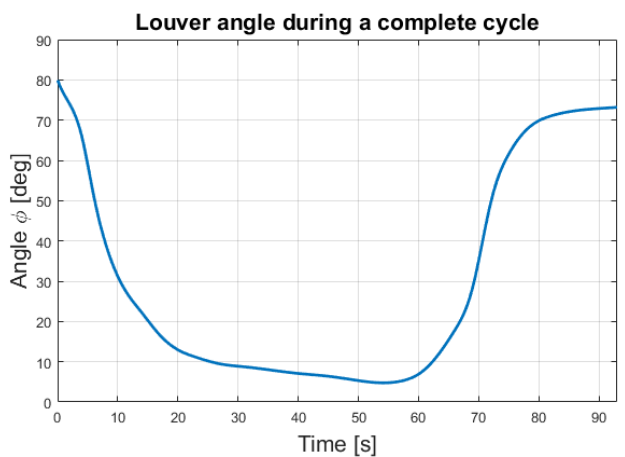

Fig. 11. The opening angle of the louver $\Phi$ over time during the first functional test under laboratory conditions.

\section{CONCLUSIONS AND FUTURE WORK}

In conclusion, the development of the subsystem proved the suitability of IRESA to operate as a spacecraft actuator, showing that a mechanical design focused on the specific features of the actuator is able to guarantee positive results immediately after the first integration process. Despite a good functioning of the first prototype, a further improvement is expected when minor issues are removed and additional tests are carried out in vacuum. A careful structural analysis combined with verifications through shaker tests are necessary in order to qualify the equipment for spaceflight; preliminary vibration tests were already carried out and successfully withstood by the prototype, but with slightly reduced loads with respect to what is specified by the most common regulations, due to limitations in the hardware at disposal.

\section{AKNOWLEDGEMENT}

We acknowledge the funding of IRESA by the Federal Ministry of Economics and Energy (BMWi), following a decision of the German Bundestag, via the German Aerospace Center (DLR) with funding grant number 50RP1750. We also want to thank the German Aerospace Center (DLR) for funding the IRESA project and the Technical University of Munich, in particular the Chair of Astronautics (LRT) of Prof. Walter for supporting us during the course of this study. Furthermore, we would like to thank the team responsible for the workshops at the TUM Campus in Garching for providing a fast and precise production of the elements to be assembled and tested.

\section{REFERENCES}

[1] P. K. Kumar and D. C. Lagoudas, Introduction to Shape Memory Alloys. In D. C. Lagoudas (Ed.), Shape Memory Alloys - Modeling and Engineering Applications. Boston, MA: Springer, 2008, pp. 3-7.

[2] V. Antonucci and A. Martone, Phenomenology of Shape Memory Alloys. In L. Lecce and A. Concilio (Ed.), Shape Memory Alloy Engineering, 1st ed. Oxford, UK: Butterworth-Heinemann (Elsevier), 2014, pp. 33-53.

[3] J. Mohd Jani, M. Leary, A. Subic, and M. A. Gibson, "A review of shape memory alloy research, applications and opportunities," in Materials \& Design (1980-2015), Volume 56, 2014, pp. 1078-1113.

[4] S. M. Dutta, F. H. Ghorbel and J. B. Dabney, "Modeling and control of a shape memory alloy actuator," in Proceedings of the 2005 IEEE International Symposium on Intelligent Control, Limassol, Cyprus, June 27-29, 2005, pp. 1007-1012.

[5] F. Schummer et al., "IRESA - Intelligent Redundant Spacecraft Actuator," in 2019 IEEE Aerospace Conference, Big Sky, MT, USA, 2019, pp. 1-12.

[6] J. E. Oberright, Spacecraft Thermal Control. In J. R. Wertz, D. F. Everett, and J. J. Puschell (Ed.), Space Mission Engineering: The New SMAD, pp. 698-699. Berlin, DE: Space Technology Library (Springer) in association w. Microcosm Press, 2011.

[7] I. Muraoka, F. L. de Sousa, F. M. Ramos, and W. R. Parisotto, "Numerical and experimental investigation of thermal louvers for space applications," in Journal of the Brazilian Society of Mechanical Sciences, 23(2), pp. 147-153.

[8] B. E. Hardt, R. D. Karam, and R. J. Eby, Louvers. In D. G. Gilmore (Ed.), Spacecraft Thermal Control Handbook, Volume I: Fundamental Technologies, 2nd ed., pp. 331-352. El Segundo, CA: The Aerospace Press, 2002.

[9] R. J. Twiggs and J. Puig-Suari, CubeSat Design Specifcation (CDS), revision 13. Available at http://www.cubesat.org/ 